\title{
Evaluación de la microcirculación sublingual en un paciente en shock séptico
}

\author{
ALEJANDRO DONOSO F. ${ }^{1,2}$, DANIELA ARRIAGADA S. ${ }^{1,2}$, PABLO CRUCES R. ${ }^{1}$, \\ JUAN ABARCA Z. ${ }^{3}$, FRANCO DÍAZ R. ${ }^{1,2}$ \\ 1. Área de Cuidados Críticos. Unidad de Gestión Clínica de Niño. Hospital Padre Hurtado, San Ramón, Santiago, Chile. \\ 2. Programa de Medicina Intensiva en Pediatría. Facultad de Medicina Clínica Alemana - Universidad del Desarrollo, Chile. \\ 3. Clínica Alemana de Santiago, Chile.
}

\begin{abstract}
Sublingual microcirculation asessment in a patient with septic shock

Introduction: Septic shock involves a complicated network of circulatory, inflammatory and metabolic disturbances,leading to cellular energetic disruption. Microcirculatory alterations are frequently observed in septic shock, being characteristic the presence of weak microcirculatory units and heterogeneous microcirculatory flow. Clinical case: A female patient, two months of age, with a pulmonary process-originated septic shock is presented. The description of microcirculation alterations at 24, 72 and 120 hrs was performed while the patient underwent therapy. A MicroScan ${ }^{\circ}$, (MicroVision Medical, Amsterdam, Holland) was utilised on the sublingual area. The patient received ventilation support, reanimation fluids, vasoactive drugs and antibiotics. The patient presented low proportion of perfused capillary vessels, low ratio of microcirculatory flow and a high heterogeneity in flow in the first measurement, all of them independant from systemic hemodynamics and disoxia indicators. These severe alterations improved progressively at 72 and 120 hrs of therapy. Discussion: Microcirculatory alterations and its time evolution may be a tool for dynamic diagnostic and severity staging assesment in septic shock. Further studies should assess microcirculation as a target for therapeutic intervention (microcirculatory resuscitation), being also of prognostic value for septic shock and severe sepsis in children.
\end{abstract}

(Key words: Microcirculation, capillary vessels, sepsis, shock).

Rev Chil Pediatr 2012; 83 (4): 377-382

\section{RESUMEN}

Introducción: El shock séptico involucra una compleja red de alteraciones circulatorias, inflamatorias y metabólicas que conducen a una disrupción energética celular. En el shock séptico se observan frecuentemente alteraciones microcirculatorias, siendo característico la existencia de unidades microcirculatorias débiles y un flujo microcirculatorio heterogéneo. Caso clínico: Se presenta una paciente de dos meses de edad con shock séptico de foco pulmonar, en la que realizamos una descripción de las alteraciones microcirculatorias a las 24, 72 y 120 h durante su tratamiento. Se utilizó MicroScan ${ }^{\circledR}$, (MicroVision Medical, Amsterdam, Holanda) en el área sublingual. La paciente recibió soporte ventilatorio, fluidos de reanimación, drogas vasoactivas y antibióticos. En la medición inicial la paciente presentaba una baja proporción de capilares perfundidos, un

Trabajo recibido el 25 de diciembre 2011, aceptado para publicación el 25 de junio de 2012.

Correspondencia a:

Dr. Alejandro Donoso F.

E-mail: adonoso@hurtadohosp.cl 
bajo índice de flujo microcirculatorio y una alta heterogeneidad de flujo, todas ellas con independencia de la hemodinamia sistémica e indicadores de disoxia. Estas alteraciones graves mejoraron progresivamente a las 72 y 120 h de tratamiento. Discusión: Las alteraciones microcirculatorias y su evolución temporal pueden ser una herramienta diagnóstica dinámica y de estratificación de gravedad en estados de shock séptico. En estudios futuros la microcirculación deberá ser evaluada como un objetivo de intervención terapéutica (resucitación microcirculatoria) presentando a su vez un rol pronóstico en el shock séptico y sepsis grave en niños.

(Palabras clave: Microcirculación, capilar, sepsis, shock).

Rev Chil Pediatr 2012; 83 (4): 377-382

\section{Introducción}

La microcirculación juega un rol fundamental en el intercambio de gases y nutrientes, adaptándose en forma constante mediante el control del tono vascular a las demandas metabólicas ${ }^{1}$.

Los parámetros macrocirculatorios del sistema cardiovascular son considerados como marcadores poco fiables de perfusión tisular y en consecuencia inadecuada para estimar el estado hemodinámico global durante condición crítica ${ }^{2}$.

Una óptima macrocirculación es un prerrequisito para una adecuada perfusión microcirculatoria; no obstante la restauración de la hemodinamia global no siempre significa que se ha logrado una adecuada perfusión regional, especialmente en condiciones de autorregulación alterada, tal como se observa en el shock ${ }^{3}$.

Las alteraciones en la perfusión microvascular, especialmente en etapa precoz del shock séptico, han sido implicadas en la falla orgánica y el Síndrome de disfunción orgánica múltiple (SDOM) $)^{4-7}$.

Actualmente la evaluación de la microcirculación es posible con las nuevas técnicas imagenológicas disponibles siendo así una herramienta de valor adicional en la monitorización del niño críticamente enfermo ${ }^{8-10}$.

Objetivo: Comunicar el caso clínico de una paciente de dos meses de edad quién curso con shock séptico y fue sometida a evaluación periódica de la microcirculación sublingual.

\section{Caso clínico}

Paciente de 2 meses de vida, sexo femenino, con antecedente de prematurez de 30 semanas por ruptura prematura de membranas y oligo- hidramnios secundario. Peso de nacimiento de 1300 g, Apgar 8-8, hospitalizada hasta las 36 semanas de edad gestacional corregida.

Historia de 24 horas de coriza, fiebre hasta $38,8^{\circ} \mathrm{C}$ y rechazo alimentario parcial.

En sus exámenes de ingreso destacaba leucopenia 5 900/ $\mathrm{mm}^{3}$, hematocrito $24 \%$, PCR 1 $\mathrm{mg} / \mathrm{l}(\mathrm{VN}:<10 \mathrm{mg} / \mathrm{l})$, gases en sangre arterial con $\mathrm{pO}_{2} 116 \mathrm{mmHg}$ y normocapnia $\left(\mathrm{pCO}_{2} 39\right.$ $\mathrm{mmHg}$ ). Previa toma de hemocultivos, se inició terapia antibiótica con cefotaxima $150 \mathrm{mg} /$ kg/día. Radiografía de tórax confirmó neumopatía basal derecha. En las horas siguientes presentó pausas respiratorias, por lo que requirió conexión a ventilación mecánica e inicio de monitorización invasiva.

Evolucionó con hipotermia, mala perfusión periférica, oliguria e hipotensión arterial que requirió reanimación con fluidos hasta $80 \mathrm{ml} /$ $\mathrm{kg}$ en las primeras 24 h, transfusión de glóbulos rojos $(15 \mathrm{ml} / \mathrm{kg})$ y uso de dopamina hasta $10 \mathrm{mcg} / \mathrm{kg} / \mathrm{min}$. Por persistencia de shock con hipotensión hasta 55/27 mmHg (presión arterial media, PAM $35 \mathrm{mmHg}$ ) se agregó norepinefrina hasta $0,2 \mathrm{mcg} / \mathrm{kg} / \mathrm{min}$ y epinefrina $0,15 \mathrm{mcg} / \mathrm{kg} / \mathrm{min}$ para obtener PAM de 55-65 $\mathrm{mmHg}$.

Evolucionó con progresión de condensación en radiografía y desarrollo de hipoxemia grave $\left(\mathrm{PaO}_{2} / \mathrm{FiO}_{2} 105 \mathrm{mmHg}\right.$ e índice de oxigenación [IO] 24), por lo que requirió conexión a ventilación de alta frecuencia oscilatoria por 48 horas desde el segundo día de ingresado (Presión media vía aérea, PMVA, $27 \mathrm{cmH}_{2} \mathrm{O}$ ).

En sus exámenes de laboratorio presentó exacerbación de leucopenia hasta $4500 / \mathrm{mm}^{3}$, sin trombocitopenia, aumento de PCR hasta 60 $\mathrm{mg} / \mathrm{l}$, hipoalbuminemia 2,5 g/dl, coagulopatía con TP 38\% e INR 2,6.

Se decidió realizar medición de la micro- 
circulación sublingual a las 24, 72 horas y al quinto día después de ingresado. (figura 1 ). Se utilizó el dispositivo MicroScan ${ }^{\circledR}$, (MicroVision Medical, Amsterdam, Holanda) según las indicaciones del fabricante y recomendaciones internacionales de consenso ${ }^{10}$.

Luego de las primeras 72 horas evolucionó favorablemente con disminución progresiva de requerimientos ventilatorios y drogas vasoactivas (figura 2). El panel para virus respiratorios fue positivo para virus respiratorio sincicial y los hemocultivos resultaron negativos. Se extubó al duodécimo día y el alta hospitalaria fue a los 27 días de ingresado.

\section{Estudio de microcirculación}

En la figura 1 se muestran imágenes representativas de la microcirculación sublingual y en la tabla 1 se muestran los parámetros macrocirculatorios, exámenes de laboratorio y soporte vasoactivo correspondiente a cada tiempo de medición. En la tabla 2 se señalan los parámetros microcirculatorios evaluados.

No encontramos diferencias importantes en la densidad capilar, sin embargo las características del flujo estaban muy alteradas en la medición inicial que mejoraron progresivamente en las mediciones sucesivas.

En la figura 1A se observan varias zonas con capilares de flujo discontinuo y ausente (flecha), reflejado en un índice de flujo microcirculatorio (MFI, Microvascular Flow Index) muy bajo, una baja proporción de capilares perfundidos
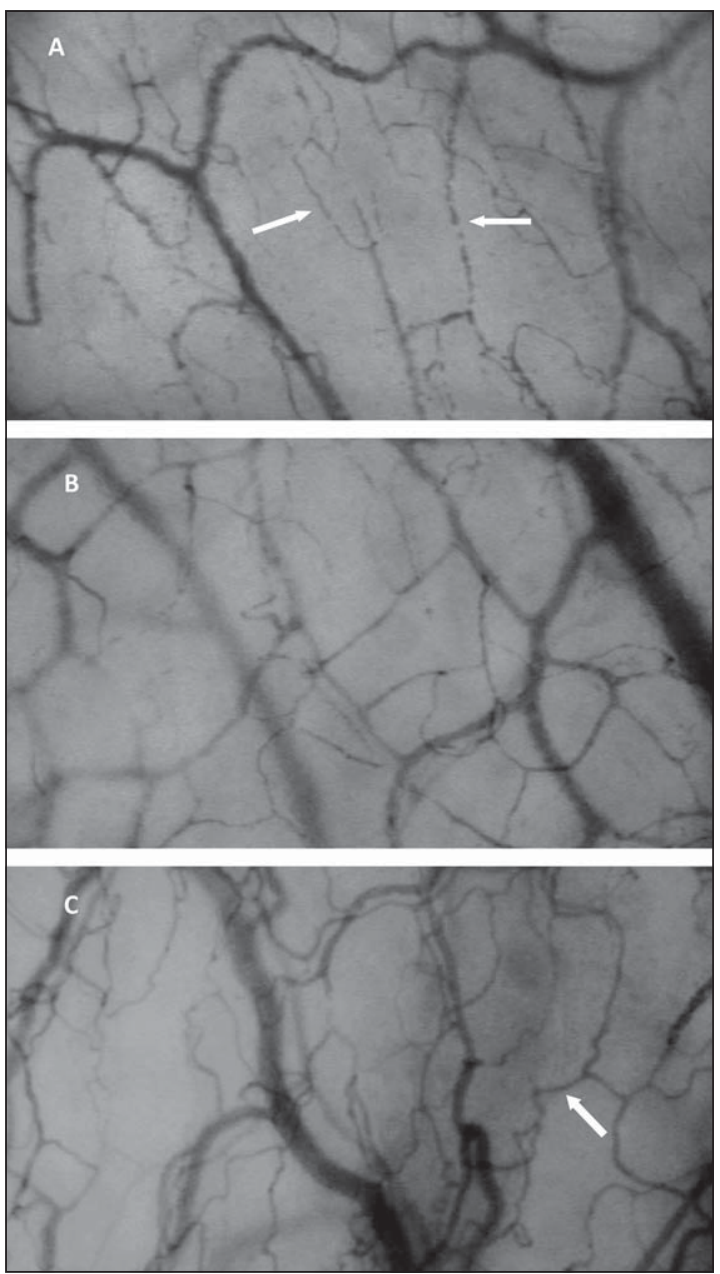

Figura 1. Imágenes representativas de la microcirculación sublingual de la paciente con shock séptico de foco pulmonar a las $24 \mathrm{~h}(\mathrm{~A}), 72 \mathrm{~h}$ (B) y $120 \mathrm{~h}$ (C) posterior al ingreso.

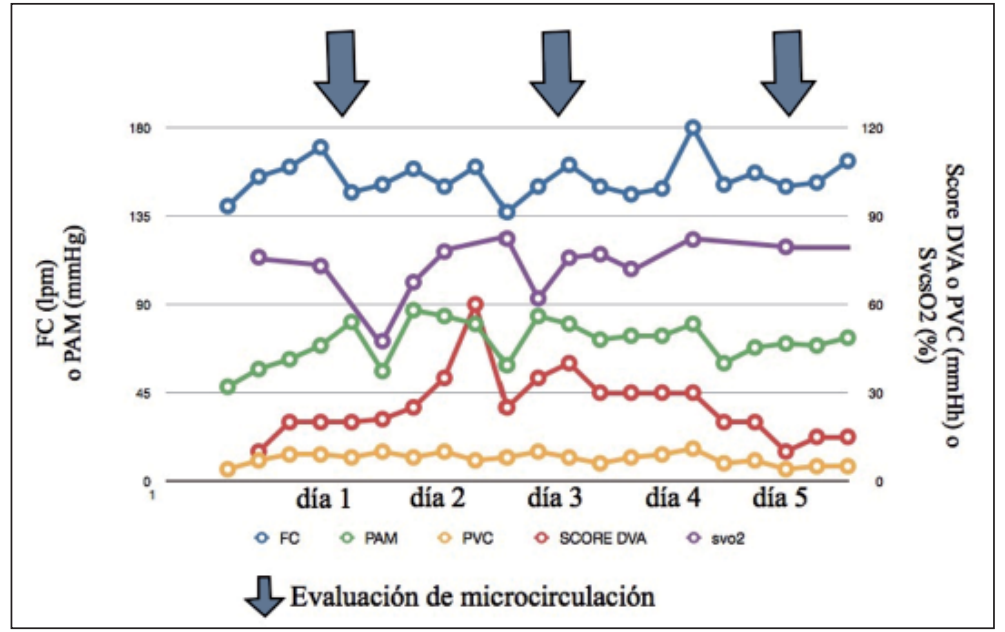

Figura 2. Evolución clínica de los principales parámetros de macrohemodinamia y requerimiento de drogas vasoactivas en la paciente cursando shock séptico. 
(PCP) y una gran heterogeneidad (Het MFI, Heterogeneity of microvascular flow index). En la figura 1B existe una mejoría cualitativa de la microcirculación, el MFI y PCP mejoró, pero aún se observan zonas con capilares con flujo alterado y muy heterogéneo.

En la figura 1C sólo se observan alteraciones menores de los capilares, acercándose a un patrón normal. Casi la totalidad de los capilares se observan bien perfundidos con una heterogeneidad muy baja.

Es importante destacar que estas alteraciones en la microcirculación no se correlacionaron con los datos macrocirculatorios y marcadores de disoxia, que fueron similares en los tres tiempos estudiados (figura 2) (tabla 1).

Tabla 1. Variables hemodinámicas, de perfusión, de laboratorio y terapéuticas en la paciente en shock séptico de foco pulmonar

\begin{tabular}{|c|c|c|c|}
\hline Día & 1 & 3 & 5 \\
\hline FC (lat/min) & 150 & 160 & 146 \\
\hline Temp $\left({ }^{\circ} \mathrm{C}\right)$ & 35,5 & 37 & 36,5 \\
\hline PAS/PAM (mmHg) & $85 / 45$ & $90 / 54$ & $85 / 50$ \\
\hline CRT Periférico (seg) & 1 & 2.3 & 1 \\
\hline $\mathrm{pH}$ & 7,31 & 7,4 & 7,43 \\
\hline $\mathrm{BE}$ & $-1,5$ & $-0,3$ & 7.6 \\
\hline $\mathrm{SVO}_{2}(\%)$ & 0,73 & 0,78 & 0,79 \\
\hline DVACO $_{2}$ & 10 & 5 & 1,4 \\
\hline $\mathrm{O}_{2} \mathrm{ER}$ & $17 \%$ & $19 \%$ & $21 \%$ \\
\hline Lactato (mmol/l) & 1,3 & 0,9 & 0,8 \\
\hline \multirow[t]{2}{*}{ Drogas Vasoactivas (mcg/kg/min) } & $\mathrm{D}(10)$ & $D(10)$ & $E(0,1)$ \\
\hline & NE (0.05) & $E(0.1)$ & \\
\hline
\end{tabular}

FC: frecuencia cardíaca; Temp: temperatura, PAS: presión arterial sistólica; PAM presión arterial media; CRT, capillary refill time, tiempo de llene capilar; $\mathrm{BE}$ : exceso de base, $\mathrm{SVO}_{2}$ : saturación venosa de oxígeno central $\mathrm{DVACO}_{2}$ : diferencia veno-arterial de $\mathrm{CO}_{2}, \mathrm{O}_{2} \mathrm{ER}$ : coeficiente de extracción de oxígeno; D: dopamina; E: epinefrina; NE: norepinefrina.

\section{Discusión}

La microcirculación corresponde a los vasos menores de 100 micrones de diámetro, es decir a la red de arteriolas, capilares y vénulas. Es ubicua y constituye la mayor superficie endotelial del cuerpo, siendo aquí donde ocurre el intercambio de oxígeno y nutrientes, remoción de $\mathrm{CO}_{2}$ y otros desechos metabólicos. Además, el endotelio presenta un papel clave en la inflamación y control de la coagulación.

Las alteraciones microcirculatorias son frecuentes de observar en el paciente críticamente enfermo, especialmente en el paciente con sepsis grave y shock séptico. Estos hallazgos son más graves en los pacientes no sobrevivientes y su persistencia juega un rol preponderante en el desarrollo de disfunción orgánica múltiple y muerte.

La falla microcirculatoria se manifiesta por una distribución anómala del flujo, con exclusión de arteriolas y capilares (shunt) ${ }^{11}$. Se puede presentar en forma independiente del estado macrocirculatorio, siendo descrita frecuentemente como causa de hipoxia celular a pesar de la normalización de los parámetros hemodinámicos ${ }^{12}$. Esto reafirma la poca validez de las metas exclusivamente hidráulicas (PAM, PVC) en la reanimación del paciente séptico. Sin embargo, siempre debemos recordar que el primer objetivo es obtener un adecuado gasto cardiaco y presión arterial antes de tratar de manipular la microcirculación.

Tabla 2. Variables microcirculatorias de la paciente en shock séptico de foco pulmonar al día 1, 3 y 5 después de ingresado

\begin{tabular}{|lccc|}
\hline Día & $\mathbf{1}$ & $\mathbf{3}$ & $\mathbf{5}$ \\
\hline Densidad capilar (vasos/mm²) & $17,3 \pm 3,1$ & $15,8 \pm 4,8$ & $18 \pm 2,2$ \\
\hline Índice de Flujo Microcirculatorio (MFI) & $0,82 \pm 0,4$ & $1,7 \pm 0,7$ & $2,7 \pm 0,3$ \\
\hline Proporción de capilares perfundidos (PCP) (\%) & $26 \pm 13$ & $45 \pm 8$ & $89 \pm 5$ \\
Densidad de capilares perfundidos (vasos/mm²) & $4,5 \pm 0,4$ & $7,1 \pm 0,7$ & $16,0 \pm 0,2$ \\
\hline Índice de Heterogeneidad de Flujo (HET MFI) & 1,37 & 1,5 & 0,4 \\
\hline
\end{tabular}

MFI: Microvascular Flow Index; PCP: proporción de capilares perfundidos; Het MFI: Heterogeneity of microvascular flow index. 
En el shock séptico se "amputa” la microcirculación, observándose diversas alteraciones como son una disminución de la densidad de capilares funcionales, un bajo flujo capilar (flujo ausente o intermitente), lo que determina un incremento de la heterogeneidad de la perfusión con capilares no perfundidos en cercanía con capilares bien perfundidos.

Múltiples mecanismos pueden explicar las alteraciones microcirculatorias descritas en la sepsis grave y/o shock séptico, estos son: disfunción endotelial, expresión de moléculas de adhesión, alteración reológica de los hematíes, rotación plaquetaria, aumento de la agregación-adhesión de leucocitos a la célula endotelial y disminución en el tamaño del glicocalix.

La alteración en la perfusión se puede sospechar ante la presencia de piel moteada, llene capilar lento o incremento de la diferencia entre temperatura central y periférica, no obstante, estos signos carecen de especificidad y sensibilidad necesaria para revelar alteraciones microcirculatorias.

El nexo entre parámetros hemodinámicos globales y la perfusión microcirculatoria es relativamente laxo. Obviamente, en un escenario de paro circulatorio, la perfusión microcirculatoria también se detiene, pero en los rangos usuales de gasto cardíaco y presión arterial, la perfusión microvascular es relativamente independiente de las variables hemodinámicas sistémicas.

Esto nos refuerza el concepto que la microcirculación no puede ser predicha, así que para una resucitación orientada en la microcirculación se debe necesariamente evaluar. La observación directa de la microcirculación es potencialmente un elemento de monitorización hemodinámica del paciente crítico pediátrico, donde otras modalidades de monitorización son especialmente limitadas.

Actualmente es empleada en clínica la videomicroscopia de luz polarizada, conocida como Sidestream Dark Field (SDF; ex-OPS). Esta técnica óptica fue desarrollada para evaluar en forma no invasiva, mediante un microscopio portátil (MicroScan ${ }^{\circledR}$, MicroVision Medical, Amsterdam, Holanda), en la cabecera del paciente ${ }^{13}$. Para este procedimiento se utiliza un diodo que emite luz verde estrobos- cópica de longitud de onda de $530 \mathrm{~nm}$. y que permite evitar las interferencias originadas por el reflejo de dicho haz de luz sobre la superficie de los órganos. Esta luz es absorbida por la hemoglobina contenida en los eritrocitos independiente de su estado de oxigenación y dispersada o reflejada por leucocitos o el resto de las estructuras, por lo que en la imagen obtenida se visualizan por contraste los glóbulos rojos fluyendo en la microcirculación (son vistos como corpúsculos grises o negros) y el resto del tejido de color claro (luz reflejada).

Las variables microcirculatorias habitualmente son evaluadas mediante dos índices aplicados en la práctica clínica. El primero fue desarrollado por De Backer et $\mathrm{al}^{12} \mathrm{y}$ se basa en el principio que la densidad microcirculatoria es proporcional al número de vasos que cruzan líneas trazadas arbitrariamente, para lo cual se dibuja un cuadriculado o rejilla de tres líneas equidistantes horizontales y verticales el cual se sobrepone en la imagen. Así se puede estimar:

a. La densidad capilar funcional, esta es igual al número de vasos que cruzan las líneas horizontales y verticales dividida por el largo total de las líneas en milímetros (n/mm) o como el largo total de los vasos dividida por el área de superficie total $\left(\mathrm{cm} / \mathrm{cm}^{2}\right)$.

b. La perfusión, la cual se describe a la visualización como presente (flujo continuo), ausente (sin flujo) o intermitente.

c. La proporción de vasos perfundidos (PPV, proportion of perfused vessels).

d. La densidad de vasos perfundidos (PVD, perfused vessel density).

El segundo indicador habitualmente ocupado es el Índice de flujo microvascular (Microvascular Flow Index, MFI) ${ }^{14}$, este nos da información del tipo de flujo en los vasos perfundidos. Es un indicador semicuantitativo que mide flujo convectivo, que permite caracterizar el tipo de flujo predominante. Se definen cuatro tipos de flujo, asignándose un número entero de 0 a 3: ausencia de flujo (0), flujo intermitente (1) definido éste como ausencia de flujo por al menos $50 \%$ del tiempo observado, flujo lento (2), flujo continuo o normal (3). Para esto cada imagen de video es dividida en 
cuatro cuadrantes iguales, promediándose todos ellos.

Otro índice que permite valorar la heterogeneidad del flujo sanguíneo es el Î́ndice de heterogeneidad de flujo microcirculatorio $(\mathrm{He}$ terogeneity of microvascular flow index, Het MFI) ${ }^{15}$. Para su cálculo se deben evaluar entre 3 a 5 sitios de la mucosa y medir el MFI en cada uno de los cuadrantes, tomando la diferencia entre el valor más elevado y el menor dividiendo este resultado por la velocidad de flujo promedio.

Conceptualmente, los tres índices (densidad capilar, velocidad y heterogeneidad del flujo) pueden contribuir independientemente a valorar el riesgo de disfunción orgánica múltiple en el paciente séptico ${ }^{16}$.

Finalmente debemos señalar que existen salvedades prácticas para un uso clínico rutinario, pues aún no se definen claramente las metas y las intervenciones terapéuticas que actúan selectivamente sobre la microcirculación ya que no han sido validadas a gran escala. Sin embargo, de ser aclarados estos aspectos, permitirán que esta modalidad de monitorización pueda orientar hacia una terapia de resucitación más completa, oportuna y efectiva en búsqueda de un adecuado reclutamiento de la microcirculación y eventualmente una mejoría en el pronóstico del paciente.

\section{Referencias}

1.- Beach JM, McGahren ED, Duling BR: Capillaries and arterioles are electrically coupled in hamster cheek pouch. Am J Physiol 1998; 275: H1489-H1496.

2.- Tibby SM, Hatherill M, Marsh MJ, Murdoch IA: Clinicians' abilities to estimate cardiac index in ventilated children and infants. Arch Dis Child 1997; 77: 516-8.

3.- $\quad$ De Backer, Ortiz JA, Salgado D: Coupling microcirculation to systemic hemodynamics. Curr Opin Crit Care
2010; 16: 250-4.

4.- Spronk PE, Zandstra DF, Ince C: Bench-to-bedside review: sepsis is a disease of the microcirculation. Crit Care 2004; 8: 462-8.

5.- Ince $C$ : The microcirculation is the motor of sepsis. Critical Care 2005; 9 (Suppl 4): S13-9.

6.- Ellis CG, Jagger J, Sharpe M: The microcirculation as a functional system. Crit Care 2005; 9 (Suppl 4): S3-S8.

7.- Abraham E, Singer M: Mechanisms of sepsis-induced organ dysfunction. Crit Care Med 2007; 35: 2408-16.

8.- De Backer D, Dubois MJ: Assessment of the microcirculatory flow in patients in the intensive care unit. Curr Opin Crit Care 2001; 7: 200-3.

9.- De Backer D, Ospina-Tascon G, Salgado D, Favory R, Creteur J, Vincent JL: Monitoring the microcirculation in the critically ill patient: current methods and future approaches. Intensive Care Med 2010; 36: 1813-25.

10.- De Backer D, Hollenberg S, Boerma C, et al: How to evaluate the microcirculation? Report of a round table conference. Critical Care 2007; 11: R101.

11.- Buwalda M, Ince C: Opening the microcirculation: can vasodilators be useful in sepsis? Intensive Care Med 2002; 28: 1208-17.

12.- De Backer D, Creteur J, Preiser JC, Dubois MJ, Vincent $J L$ : Microvascular blood flow is altered in patients with sepsis. Am J Respir Crit Care Med 2002; 166: 98-104.

13.- Ince C: Sidestream dark field (SDF) imaging: an improved technique to observe sublingual microcirculation. Crit Care 2005; 8 (Suppl 1): P72.

14.- Spronk PE, Ince C, Gardien MJ, Mathura KR, Oudemans-van Straaten HM, Zandstra DF: Nitroglycerin in septic shock after intravascular volume resuscitation. Lancet 2002; 360: 1395-6.

15.- Trzeciak S, Dellinger RP, Parrillo JE, et al: Early microcirculatory perfusion derangements in patients with severe sepsis and septic shock: relationship to hemodynamics, oxygen transport, and survival. Ann Emerg Med 2007; 49: 88-98.

16.- Vincent JL, De Backer D: Microvascular dysfunction as a cause of organ dysfunction in severe sepsis. Crit Care 2005; 9 Suppl 4: S9-12. 